\title{
Skin acceptability of a cosmetic moisturizer formulation in female subjects with sensitive skin
}

\section{Stephanie J Nisbet}

Skin Health Medical Affairs, GSK Consumer Healthcare, Weybridge, Surrey, UK
Correspondence: Stephanie J Nisbet Skin Health Research \& Development. GSK Consumer Healthcare, St George's Avenue, Surrey, Weybridge KTI 3 ODE, UK

$\mathrm{Tel}+44$ I932822 40I

$\mathrm{Fax}+441932822 \quad 100$

Email Stephanie.J.Nisbet@gsk.com
This article was published in the following Dove Press journal: Clinical, Cosmetic and Investigational Dermatology

Purpose: This 3-week, open-label, noncomparative clinical study evaluated the skin acceptability of a cosmetic moisturizer in subjects with sensitive skin, by monitoring adverse events (AEs) and cutaneous discomfort related to normal usage.

Materials and methods: Female subjects aged between 18-60 years, with Fitzpatrick phototype classification I-IV and sensitive skin, verified by a positive reaction on the stinging test at screening, were included. Subjects applied the moisturizer to their face and body twice daily for $21 \pm 2$ days at home and recorded study product usage and feelings of cutaneous discomfort (eg, dryness, prickling, stinging, and itching) in a diary; any AEs were reported to the clinic. At study end, skin acceptability of the moisturizer was investigator-assessed based on the nature of AEs and subjects' self-reported feelings of discomfort, and by clinical evaluation of skin reactions in the area of moisturizer application (appearance of erythema, formation of edema, and skin desquamation; scored according to an adapted Draize and Kligman scale). Only subjects with a treatment compliance of $\geq 80 \%$ were included in the final analysis.

Results: In total, 35 subjects initiated and completed the study; all were compliant to the minimum study product usage. Per investigator clinical dermatological assessment at study end, none of the 35 subjects had skin reactions in the area of moisturizer application and there were no reported AEs. One subject reported sensations of mild prickling and itching immediately after applying the moisturizer (not classified as AEs), which spontaneously remitted after complete absorption of the product and were noted only in exposed areas. These events were considered by the investigator as being possibly/probably related to the use of study product; however, no clinical signs of skin reaction were observed in the exposed areas.

Conclusion: This cosmetic moisturizer appears generally well tolerated and suitable for topical use in subjects with sensitive skin.

Keywords: skin barrier, safety testing, sensitive skin, skin acceptability, irritation

\section{Introduction}

Sensitive skin is a condition of subjective cutaneous hyperreactivity to environmental conditions. ${ }^{1}$ Subjects with sensitive skin may experience a noninflammatory response to products applied topically to the skin, characterized by sensorial reactions such as stinging, burning, or itching, in the absence of visible irritation-related skin changes or an active immune response/allergic reaction. ${ }^{2-4}$ Sensitive skin may be more prone to adverse reactions to cosmetic products, ${ }^{4}$ including irritation, burning sensation, redness, pruritus, and erythema at the product application site. ${ }^{5}$ Substances known to trigger an irritation response in sensitive skin include benzoic acid, cinnamic acid, nonionic emulsifiers, sodium laurel sulfate, bronopol, lactic acid, propylene glycol, urea, and 
sorbic acid. ${ }^{6,7}$ It is important, therefore, that cosmetic skin products are clinically evaluated under normal conditions of use and in the subject population for whom they are intended, prior to approval for use.

Acceptability trials aim to confirm the absence of risk of primary and/or cumulative irritation and to identify feelings of discomfort associated with cosmetic product application under normal or reasonably predictable conditions of use. Prior to clinical testing, the cosmetic moisturizer was assessed for predicted tolerability based on the toxicological profiles of the ingredients. At the concentrations of use in the formulation, none of the ingredients were considered to be skin irritants or skin sensitisers. In vitro testing using established models confirmed the predicted irritancy potential of this formulation (data not shown). The present study was conducted to evaluate the acceptability of a cosmetic moisturizer in female subjects with sensitive skin under normal conditions of use.

\section{Materials and methods Study design}

This was a 3-week, open-label, noncomparative clinical study conducted to evaluate a cosmetic moisturizer in female subjects with sensitive skin (GSK study 205024). The overall objective was to verify the acceptability of the moisturizer on sensitive skin through monitoring of adverse events (AEs) and cutaneous discomfort related to its application under normal conditions of use.

The moisturizer evaluated in this study contained the following ingredients: aqua, Butyrospermum parkii butter, caprylic/capric triglyceride, carbomer, ceramide 3, Cocos nucifera oil, glycerin, hydrogenated lecithin, hydroxyethyl cellulose, pentylene glycol, sodium carbomer, squalane, and xanthan gum.

The study was conducted between January 11 and February 3, 2016, at a single center in Vila Martina, Valinhos, Brazil.

\section{Subjects}

Eligible subjects were selected to reflect the target population for this product and were female, aged 18-60 years, with Fitzpatrick phototype classification I-IV, and sensitive skin verified by a positive stinging test ${ }^{8}$ at screening.

Subjects were excluded if they had any of the following: skin marks in the product application area; active dermatoses (local or disseminated); a history of allergic reactions to topical cosmetics or drugs; immunodeficiency; intense exposure to sunlight or tanning sessions within 15 days prior to screening or during the study; aesthetic and/or dermatological treatment in the product application area within 3 weeks prior to screening or during the study; vaccination within 3 weeks prior to screening or during the study; therapeutic, topical, or systemic use of immunosuppressants, antihistamines, nonsteroidal anti-inflammatory drugs, and corticosteroids within 2 weeks prior to screening (within 30 days in the case of systemic corticosteroids); and oral or topical treatment with vitamin A acid and/or its derivatives within 1 month of screening or during the study.

Subjects were asked to avoid the following activities during the study: applying nonstudy products to the skin test area; changing dietary habits, cosmetic habits (including hygiene products) or hormonal treatment(s); skin cleansing, exfoliating, or applying other aesthetic treatments in the product test area; exposing skin to excessive sunlight and/ or using artificial tanning beds; and using protocol-restricted concomitant medications.

The study was conducted in accordance with the ethical principles founded in the Declaration of Helsinki and Good Clinical Practice Guidelines and was approved by the Research Ethics Committee of the Faculdade de Medicina de Jundiai (Reference number: 48430115.2.0000.5412). All subjects provided written informed consent.

\section{Study procedures and assessments}

The study comprised 2 scheduled clinic visits. At Visit 1 (day 1), initial dermatological assessments were conducted and subjects were screened for eligibility against the inclusion/exclusion criteria. Eligible subjects underwent a stinging test, which comprised application of $10 \%$ lactic acid to the nasolabial fold (negative control was saline applied to the contralateral fold) and subsequent subject grading of the intensity of the reaction induced (prickling, itching, burning sensation, stinging sensation, numbness) according to a 4 -point scale $(0=$ no sensation, $1=$ mild sensation, $2=$ moderate sensation, and $3=$ severe sensation). ${ }^{8}$ Subjects with a selfassessed reaction of intensity $\geq 2$ (moderate/severe intensity) were considered to have a positive result for sensitive skin and were included in the study.

Subjects were provided with a preweighed bottle of the cosmetic moisturizer for use at home and were instructed to apply the product to skin on the face and body twice daily, every day for $21 \pm 2$ days. A diary was also provided to subjects in which to record their product usage and to note any feelings of cutaneous discomfort experienced or other complaints/comments, including the dates the subject should return to the study site. Subjects were instructed to report to the clinic if they experienced AEs at any time. At the end of the study period, subjects returned 
to the clinic (Visit 2; day 21 \pm 2 ) for investigator review of the product usage diary, assessment of treatment compliance, and final clinical dermatological assessments.

Study product acceptability was investigator-assessed based on the nature of AEs and feelings of cutaneous discomfort reported by subjects and by clinical evaluation of signs of skin reaction. AEs were classed as any medical occurrence, regardless of a causal relationship to the study product, including any expected symptoms, abnormal laboratory results, or concomitant illnesses. Feelings of cutaneous discomfort (dryness, prickling, stinging, itching, or other sensations) were coded by subjects in the product usage diary as either mild or moderate-to-intense in severity and sensations persisting for $>15$ minutes were noted; however, these feelings/sensations were not classed as AEs unless considered clinically significant by the study physician. Skin reactions (appearance of erythema, formation of edema, and skin desquamation) in the area of study product application were scored by the investigator according to an adapted Draize and Kligman scale (Table S1), ${ }^{9,10}$ and the overall reaction intensity was classified according to the sum of the individual scores. A total score of 0-2 was classified as no reaction, 3-4 a mild reaction, 5-8 a moderate reaction, and $>8$ an intense reaction. Skin reactions were classified as AEs in this study. To be considered compliant with study product use, subjects must have applied the product at least $80 \%$ of the instructed number of times. Only data from subjects compliant with study product use were used in the evaluation of local tolerance. As the product was to be used at least twice a day, for a minimum of 19 days, a minimum of 38 applications were expected. Therefore, each subject must have used the test product a minimum of 30 times in order to be included in the final analysis. All subjects were compliant with study product use.

\section{Statistical considerations}

Thirty-five subjects were planned for inclusion, to ensure that at least 30 would complete the study. For analysis of product acceptability, only data from subjects with a minimum treatment compliance of $80 \%$ were considered. As the study product was to be used twice daily for at least 19 days, a minimum of 38 applications were expected per subject. To meet the $\geq 80 \%$ compliance criterion, each subject was required to use the study product at least 30 times to be included in the final analysis.

\section{Results}

Thirty-six female subjects were screened for eligibility; of these, 35 subjects initiated and completed the study. All subjects were compliant to the minimum study product usage and were included in the final analysis of product acceptability.

None of the 35 subjects experienced AEs during the study. Per investigator clinical dermatological assessment at study end, none of the subjects had skin reactions in the area of study product application (Table 1). One subject reported experiencing sensations of mild prickling and mild itching immediately after study product application over 22 consecutive days, but these were not classified as AEs (Table 1). These sensations were reported by the subject to have spontaneously remitted after complete absorption of the product (maximum 10 minutes) and were noted only in exposed areas. These events were considered by the investigator as being possibly/ probably related to study product use; however, no clinical signs of skin reaction were observed in the exposed areas.

\section{Discussion}

The benefits of moisturizers containing shea butter have been studied in atopic dermatitis/eczema populations. ${ }^{11,12}$ The clinical efficacy and acceptability of a shea butter-containing moisturizer was investigated in pediatric patients with atopic dermatitis over 4 weeks and reported general acceptability as "very good" or "good" in 74\% of the 34 patients recruited. This group demonstrated a significant reduction in mean pruritus score and a significant mean improvement in Children's Dermatology Life Quality index. ${ }^{11}$ In another study, an over-the-counter shea butter-containing moisturizer was shown to significantly improve the signs and symptoms of mild-to-moderate body eczema in adults after 2 weeks. ${ }^{12}$

In this study, the study population differed from those previously reported, ${ }^{11,12}$ and the acceptability of a cosmetic shea

Table I Skin reactions and reports of feelings of cutaneous discomfort (study population, $\mathrm{N}=35$ )

\begin{tabular}{ll}
\hline $\begin{array}{l}\text { Skin reactions and feelings of cutaneous } \\
\text { discomfort }\end{array}$ & Subjects, $\mathbf{n}(\%)$ \\
\hline Skin reaction classification & \\
No reaction & $35(100)$ \\
Equivocal & 0 \\
$\quad$ Reaction without causal relationship & 0 \\
Feelings of cutaneous discomfort & \\
Dryness & \\
Prickling & 0 \\
Stinging & I $(2.9)^{c, d}$ \\
Itching & 0 \\
Other sensations & I $(2.9)^{c, d}$ \\
\hline
\end{tabular}

Notes: aPer investigator assessment (skin reactions were classified as AEs); ' selfreported in subject's product usage diary (feelings of cutaneous discomfort were

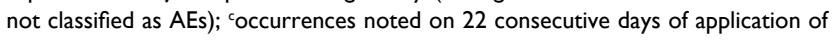
the study product; ${ }^{d}$ noted by the subject as mild in intensity.

Abbreviation: $A E$, adverse event. 
butter-containing moisturizer was investigated in subjects with clinically assessed sensitive skin. These data suggest that the evaluated cosmetic moisturizer was generally well tolerated in female subjects with sensitive skin. Based on subjects' self-recorded assessments of feelings of discomfort during the study period and investigator's clinical dermatological assessments obtained following $21 \pm 2$ days of use of the study product, it was verified that no AEs or skin reactions occurred in the 35 participants. The exclusion of subjects with a history of allergic reactions to topical cosmetics or drugs from the study also lends support to the acceptability of the cosmetic moisturizer in the target population with sensitive skin. The study results also concurred with the predicted tolerability profile of the formulation based on the ingredient evaluation and in vitro tests.

In interpreting these data, several limitations of the analysis should be considered, including the small sample size, conduction of the study at a single center, evaluation of skin acceptability of the study product exclusively in female subjects, the subjective nature of the method for assessing cutaneous discomfort after study product application (subjects' threshold levels for severity of discomfort may differ), and a reliance on subjects to accurately record/code in their product usage diaries.

\section{Conclusion}

Based on the findings of this acceptability test, this cosmetic moisturizer appears generally well tolerated and suitable for topical use in subjects with sensitive skin.

\section{Data sharing statement}

Data supporting the results presented in this manuscript can be found on the GSK clinical study register (GSK study 205024; https://www.gsk-clinicalstudyregister.com/ search/?search_terms $=205024$ ) and can be requested.

\section{Acknowledgments}

The author would like to thank all subjects for their participation in this study. The cosmetic moisturizer evaluated in this study was supplied by Stiefel Laboratories (Ireland) Limited, Sligo, Ireland. Editorial support (in the form of writing assistance, assembling tables, collating author comments, grammatical editing, and referencing) was provided by Emma Landers, PhD, of Gardiner-Caldwell Communica- tions, Macclesfield, UK, and was funded by GSK Consumer Healthcare.

This study was sponsored by GSK Brasil Ltda (GSK study 205024); the sponsor was responsible for oversight of study design, study conduct, and the clinical study report. The legal registered sponsor of this study was GSK Research \& Development Limited (Brentford, Middlesex, UK); employees of the legal sponsor were involved in data analysis/interpretation and manuscript writing/review.

This work has not previously been presented in part or in full.

\section{Disclosure}

Stephanie J Nisbet discloses employment with GSK Consumer Healthcare and stock/share ownership in GSK. The author reports no other conflicts of interest in this work.

\section{References}

1. Berardesca E, Farage M, Maibach H. Sensitive skin: an overview. Int J Cosmet Sci. 2013;35(1):2-8.

2. Coverly J, Peters L, Whittle E, Basketter DA. Susceptibility to skin stinging, non-immunologic contact urticaria and acute skin irritation; is there a relationship? Contact Dermatitis. 1998;38(2):90-95.

3. Pons-Guiraud A. Sensitive skin: a complex and multifactorial syndrome. J Cosmet Dermatol. 2004;3(3):145-148.

4. Farage MA, Katsarou A, Maibach HI. Sensory, clinical and physiological factors in sensitive skin. Contact Dermatitis. 2006;55(1):1-14.

5. Lumelsky VJ, Shur MS, Wagner S. Sensitive skin. IEEE Sensors J. 2001;1(1):41-51.

6. de Groot AC, White IR, Flyvholm MA, Lensen G, Coenraads PJ. Formaldehyde-releasers in cosmetics: relationship to formaldehyde contact allergy. Part 1. Characterization, frequency and relevance of sensitization, and frequency of use in cosmetics. Contact Dermatitis. 2010;62(1):2-17.

7. Lundov MD, Johansen JD, Carlsen BC, Engkilde K, Menné T, Thyssen JP. Formaldehyde exposure and patterns of concomitant contact allergy to formaldehyde and formaldehyde-releasers. Contact Dermatitis. 2010;63(1):31-36.

8. Christensen M, Kligman AM. An improved procedure for conducting lactic acid stinging tests on facial skin. J Soc Cosmetic Chem. 1996;47:1-11.

9. Draize JH, Woodard G, Calvery HO. Methods for the study of irritation and toxicity of substances applied topically to the skin and mucous membranes. J Pharmacol Exp Ther. 1944;82(3):377-390.

10. Draize JH. Dermal toxicity. Appraisal of Chemicals in Food, Drugs and Cosmetics. 3rd ed. Austin, TX: Association of Food and Drug Officials of the United States; 1975:46-59.

11. Hon KL, Tsang YC, Pong NH, et al. Patient acceptability, efficacy, and skin biophysiology of a cream and cleanser containing lipid complex with shea butter extract versus a ceramide product for eczema. Hong Kong Med J. 2015;21(5):417-425.

12. Draelos ZD. A pilot study investigating the efficacy of botanical antiinflammatory agents in an OTC eczema therapy. J Cosmet Dermatol. 2016;15(2):117-119. 


\section{Supplementary material}

Table SI Skin reaction assessment criteria

\begin{tabular}{ll}
\hline Score & Appearance of erythema \\
\hline 0 & None \\
1 & Very mild erythema \\
2 & Well-defined erythema \\
3 & Moderate erythema \\
4 & Severe erythema \\
\hline Score & Formation of edema \\
\hline 0 & None \\
\hline & Very mild edema (almost perceptible) \\
2 & Mild edema (defined area, beginning of swelling) \\
3 & Moderate edema (swelling of $\sim$ I mm) \\
4 & Intense edema (growth $>$ I mm and beyond application area) \\
\hline Score & Skin desquamation \\
\hline 0 & None \\
\hline & Dryness \\
2 & Thin scales \\
3 & Moderate scales \\
4 & Large scales \\
\hline Score & Overall skin reaction classification \\
\hline $0-2$ & No reaction/doubtful \\
$3-4$ & Mild reaction \\
$5-8$ & Moderate reaction \\
$>8$ & Intense reaction \\
\hline Notes: & \\
\hline
\end{tabular}

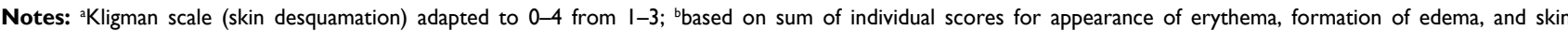
desquamation. Skin reaction assessment criteria adapted from Draize et al' and Draize. ${ }^{2}$

\section{References}

1. Draize JH, Woodard G, Calvery HO. Methods for the study of irritation and toxicity of substances applied topically to the skin and mucous membranes. J Pharmacol Exp Ther. 1944;82(3):377-390.

2. Draize JH. Dermal toxicity. Appraisal of Chemicals in Food, Drugs and Cosmetics. 3rd ed. Austin, TX: Association of Food and Drug Officials of the United States; 1975:46-59.

\section{Publish your work in this journal}

Clinical, Cosmetic and Investigational Dermatology is an international, peer-reviewed, open access, online journal that focuses on the latest clinical and experimental research in all aspects of skin disease and cosmetic interventions. This journal is included on PubMed. The manuscript management system is completely online and includes a very quick and fair peer-review system, which is all easy to use. Visit http://www.dovepress.com/testimonials.php to read real quotes from published authors

Submit your manuscript here: https://www.dovepress.com/clinical-cosmetic-and-investigational-dermatology-journal 